\title{
On Hyperquasigroups
}

\author{
JANEZ UŠAN AND RADOSLAV GaLiĆ
}

ABSTRACT. In the paper we define and study hyperquasigroups of the rang $m(\in N)$.

\section{Preliminaries}

Definition 1.1. Let $Q$ be a non-empty set and $P(Q)$ its power set. Let $A$ be a mapping of the set $Q^{2}$ into the set $P(Q)$. Then:

a) we say that the mapping $A$ is a hyperoperation in $Q$; and

b) we say that the ordered pair $(Q ; A)$ is a hypergroupoid.

Definition 1.2. Let $(Q ; A)$ be a hypergroupoid. Also, let:

$$
\mathbf{A}(X, Y) \stackrel{\text { def }}{=} \begin{cases}\bigcup_{(x, y) \in X \times Y} A(x, y) ; & X \neq \emptyset, Y \neq \emptyset \\ \emptyset ; & X=\emptyset \text { or } Y=\emptyset\end{cases}
$$

for all $X, Y \in P(Q)$. Then, we say that the groupoid $(P(Q) ; \mathbf{A})$ is a associated (or coresponds) to the hypergroupoid $(Q ; A)$. (For example: Table 1 and Table 2.)

\begin{tabular}{c|c|c|}
$A$ & 1 & 2 \\
\hline 1 & $\{1\}$ & $\{2\}$ \\
\hline 2 & $\{2\}$ & $\{1,2\}$ \\
\hline
\end{tabular}

Table 1

\begin{tabular}{c|c|c|c|c}
$\mathbf{A}$ & $\{1\}$ & $\{2\}$ & $\{1,2\}$ & $\emptyset$ \\
\hline$\{1\}$ & $\{1\}$ & $\{2\}$ & $\{1,2\}$ & $\emptyset$ \\
\hline$\{2\}$ & $\{2\}$ & $\{1,2\}$ & $\{1,2\}$ & $\emptyset$ \\
\hline$\{1,2\}$ & $\{1,2\}$ & $\{1,2\}$ & $\{1,2\}$ & $\emptyset$ \\
\hline$\emptyset$ & $\emptyset$ & $\emptyset$ & $\emptyset$ & $\emptyset$ \\
\hline
\end{tabular}

Table 2

Remark 1.1. a) If for all $x, y \in Q A(x, y) \in \mathbf{Q}$, where $\mathbf{Q} \stackrel{\text { def }}{=}\{\{x\} \mid x \in Q\}$, then $(\mathbf{Q}, \mathbf{A})$ is a groupoid.

b) If $(Q, \mathcal{A})$ is a groupoid and $A(x, y) \stackrel{\text { def }}{=}\{\mathcal{A}(x, y)\}$, then $(Q ; A)$ is a hypergroupoid.

2000 Mathematics Subject Classification. 20N99, 20N05.

Key words and phrases. Quasigroups, hypergroupoids, hyperquasigroups. 
c) Let $\mathcal{A}: D \rightarrow Q, D \subseteq Q^{2}$. [i.e. $(Q ; \mathcal{A})$ is a partial groupoid] and let

$$
A(x, y) \stackrel{\text { def }}{=} \begin{cases}\{\mathcal{A}(x, y) ;\} ; & (x, y) \in D \\ \emptyset ; & (x, y) \in Q^{2} \backslash D\end{cases}
$$

for all $x, y \in Q .{ }^{1}$ Then $(Q ; A)$ is a hypergroupoid.

d) Let $\rho$ be a 3 -ary relation in $Q$. Also, let

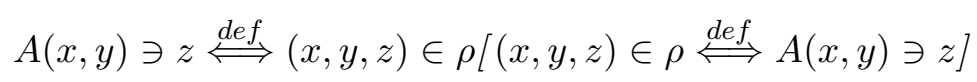

for all $x, y, z \in Q$. Then $(Q ; A)$ is a hypergroupoid /then $\rho$ is a 3-ary realtion in $Q]$.

Definition 1.3. Let $(Q ; A)$ be a hypergroupoid and let for all $(x, y) \in Q^{2} \quad A(x, y) \neq$ $\emptyset$. Then, we say that $(Q, A)$ is a hypergroup iff the following statements hold:

(a) $\mathbf{A}(\mathbf{A}(\{x\},\{y\}),\{z\})=\mathbf{A}(\{x\}, \mathbf{A}(\{y\}),\{z\}))$ for each $x, y, z \in Q$; and

(b) For every $a, b \in Q$ there is at least one $x \in Q$ and at least one $y \in Q$ such that the following formulas hold

$$
A(a, x) \ni b \quad \text { and } \quad A(y, a) \ni b .
$$

Remark 1.2. A notion of a hypergroup was introduced by F. Marty in [1] as a generalization of the notion of a group. Cf. [2].

\section{PARAStrophic hyperoperations}

Proposition 2.1. Let $(Q ; A)$ be a hypergroupoid and let $\alpha$ be a permutation of the set $\{1,2,3\}$. Also, let

$$
A^{\alpha}\left(x_{1}, x_{2}\right) \ni x_{3} \stackrel{\text { def }}{\Leftrightarrow} A\left(x_{\alpha(1)}, x_{\alpha(2)}\right) \ni x_{\alpha(3)}
$$

for all $x_{1}, x_{2}, x_{3} \in Q$. Then $\left(Q, A^{\alpha}\right)$ is a hypergroupoid.

Proof. By Def. 1.1.

Definition 2.1. Let $(Q ; A)$ be a hypergroupoid and let $\alpha$ be a permutation of the set $\{1,2,3\}$. Also, let

$$
A^{\alpha}\left(x_{1}, x_{2}\right) \ni x_{3} \stackrel{\text { def }}{\Leftrightarrow} A\left(x_{\alpha(1)}, x_{\alpha(2)}\right) \ni x_{\alpha(3)}
$$

\footnotetext{
${ }^{1}$ If $D=\emptyset$, then $A(x, y)=\emptyset$ for all $(x, y) \in Q^{2}$.
} 
for all $x_{1}, x_{2}, x_{3} \in Q$. Then, we shall say that the hyperoperation $A^{\alpha}$ is a $\alpha$-parastrophic hyperoperation to the hyperoperation $A$. Let:

(a)

$$
\begin{gathered}
A \stackrel{I \text { def }}{=} A[I=\{(x, x) \mid x \in\{1,2,3\}\}], \\
{ }^{-1} A\left(x_{1}, x_{2}\right) \ni x_{3} \stackrel{\text { def }}{\Leftrightarrow} A\left(x_{3}, x_{2}\right) \ni x_{1}, \\
A^{-1}\left(x_{1}, x_{2}\right) \ni x_{3} \stackrel{\text { def }}{\Leftrightarrow} A\left(x_{1}, x_{3}\right) \ni x_{2}, \\
A^{*}\left(x_{1}, x_{2}\right) \ni x_{3} \stackrel{\text { def }}{\Leftrightarrow} A\left(x_{2}, x_{1}\right) \ni x_{3} \quad\left[A^{*}\left(x_{1}, x_{2}\right)=A\left(x_{2}, x_{1}\right)\right], \\
-1\left(A^{*}\right)\left(x_{1}, x_{2}\right) \ni x_{3} \stackrel{\text { def }}{\Leftrightarrow} A\left(x_{3}, x_{1}\right) \ni x_{2} \text { and } \\
\left(A^{*}\right)^{-1}\left(x_{1}, x_{2}\right) \ni x_{3} \stackrel{\text { def }}{\Leftrightarrow} A\left(x_{2}, x_{3}\right) \ni x_{1}
\end{gathered}
$$

for all $x_{1}, x_{2}, x_{3} \in Q$. (See, also [4] or [6])

Proposition 2.2. Let $(Q ; A)$ be a hypergroupoid and let $\alpha$ be a permutation of the set $\{1,2,3\}$. Then

$$
\begin{gathered}
\left\{A^{\alpha},{ }^{-1}\left(A^{\alpha}\right),\left(A^{\alpha}\right)^{-1},\left(A^{\alpha}\right)^{*},{ }^{-1}\left(\left(A^{\alpha}\right)^{*}\right),\left(\left(A^{\alpha}\right)^{*}\right)^{-1}\right\}= \\
\left\{A,{ }^{-1} A, A^{-1}, A^{*},{ }^{-1}\left(A^{*}\right),\left(A^{*}\right)^{-1}\right\} .
\end{gathered}
$$

Proof. By $(a)$ and since $(\{1,2,3\} !, \circ)$ is a group.

\section{Hypergroupoids WiTH DIVISIONS}

Definition 3.1. We shall say that a hypergroupoid $(Q ; A)$ is a hypergroupoid with divisions iff the following statemets hold:

(1) For all $a, b \in Q \quad A(a, b) \neq \emptyset$,

(2) For all $a, b \in Q \quad{ }^{-1} A(a, b) \neq \emptyset,^{2}$

(3) For all $a, b \in Q A^{-1}(a, b) \neq \emptyset .^{3}$

Cf.1.4 and 1.5.

Proposition 3.1. Let $(Q ; A)$ be a hypergroupoid with division. Then, for all $\alpha \in\{1,2,3\}$ ! the hypergroupoid $\left(Q, A^{\alpha}\right)$ is a hypergroupoid with divisions.

Proof.

a) By Def. 2.2, (a) from 2, we obtain

$$
\begin{aligned}
A^{*}\left(x_{1}, x_{2}\right) & =A\left(x_{2}, x_{1}\right), \\
-1\left(A^{*}\right)\left(x_{1}, x_{2}\right) & =A^{-1}\left(x_{2}, x_{1}\right) \quad \text { and } \\
\left(A^{*}\right)^{-1}\left(x_{1}, x_{2}\right) & ={ }^{-1} A\left(x_{2}, x_{1}\right)
\end{aligned}
$$

for all $x_{1}, x_{2} \in Q$. Whence, by (1)-(3) from Def. 3.1, we conclude the statements

(4) For all $a, b \in Q \quad A^{*}(a, b) \neq \emptyset$,

${ }^{2}$ For all $a, b \in Q$ there is at least one $x \in Q$ such that the following formula holds $A(x, a) \ni b$.

${ }^{3}$ For every $a, b \in Q$ there is at least one $y \in Q$ such that the following formula holds $A(a, y) \ni b$. 
(5) For all $a, b \in Q^{-1}\left(A^{*}\right)(a, b) \neq \emptyset$ and

(6) For all $a, b \in Q\left(A^{*}\right)^{-1}(a, b) \neq \emptyset$

hold.

b) By a) and by Prop. 2.3, we conclude that the proposition is satisfied.

\section{HyPERCANCELATION HYPERgROUPOIDS}

Definition 4.1. We shall say that a hypergroupoid $(Q ; A)$ is a hypercancellation hypergroupoid iff there are $p, q, r \in N \cup\{0\}$ such that the following statements hold:

(i) $\left(\exists\left(a_{1}, a_{2}\right) \in Q^{2}\right)\left|A\left(a_{1}^{2}\right)\right|=p$ and $\left(\forall(x, y) \in Q^{2}\right)|A(x, y)| \leq p$;

(ii) $\left.\left(\exists\left(b_{1}, b_{2}\right) \in Q^{2}\right)\right|^{-1} A\left(b_{1}^{2}\right) \mid=q$ and $\left.\left(\forall(x, y) \in Q^{2}\right)\right|^{-1} A(x, y) \mid \leq q$; and

(iii) $\left(\exists\left(c_{1}, c_{2}\right) \in Q^{2}\right)\left|A^{-1}\left(c_{1}^{2}\right)\right|=r$ and $\left(\forall(x, y) \in Q^{2}\right)\left|A^{-1}(x, y)\right| \leq r$.

Moreover, we shall say that a hypercancellation hypergroupoid $(Q ; A)$ is a hypercancelation hypergroupoid of the type $\langle p, q, r\rangle$.

Example 4.1. Let $(Q ; \mathcal{A})$ be a cancellation groupoid. Then $(Q ; A)$, where

$$
A(x, y) \stackrel{\text { def }}{=}\{\mathcal{A}(x, y)\}
$$

for all $(x, y) \in Q^{2}$, is a hypercancellation hypergroupoid of the type $\langle 1,1,1\rangle$.

Example 4.2. Let $Q$ be a non-empty set and let $A(x, y)=\emptyset$ for all $(x, y) \in Q^{2}$. Then:

a) ${ }^{-1} A(x, y)=\emptyset$ for all $(x, y) \in Q^{2}$;

b) $A^{-1}(x, y)=\emptyset$ for all $(x, y) \in Q^{2}$; and

c) $(Q, A)$ is a hypercancellation hypergroupoid of the type $\langle 0,0,0\rangle$.

Example 4.3. Let $Z$ be the set of all integers and let

$$
\mathcal{A}(2 k-1,2 t-1)=\mathcal{A}(2 k-1,2 t)=\mathcal{A}(2 k, 2 t-1)=\mathcal{A}(2 k, 2 t)=k+t
$$

for all $k, t \in Z$ Also, let

$$
A(x, y) \stackrel{\text { def }}{=}\{\mathcal{A}(x, y)\}
$$

for all $x, y \in Z$. Then $(Q ; A)$ is a hypercancellation hypergroupoid of the type $<1,2,2>$.

Theorem 4.1. Let $(Q ; A)$ be a hypercancellation hypergroupoid of the type < $p, q, r>$. Then ${ }^{-1} A, A^{-1}, A^{*},{ }^{-1}\left(A^{*}\right)$ and $\left(A^{*}\right)^{-1}$ are hypercancellation hyperoperations, respectively, of the type $\langle q, p, r\rangle,\langle r, q, p\rangle,\langle p, r, q\rangle,\langle r, p, q\rangle$ and $<q, r, p>$

Sketch of a part of the proof.

$$
\begin{aligned}
{ }^{-1}\left({ }^{-1} A\right)=A, & \\
\left(^{-1} A\right)^{-1}(x, y) \ni z & \Leftrightarrow{ }^{-1} A(x, z) \ni y \\
& \Leftrightarrow A(y, z) \ni x \\
& \Leftrightarrow A^{-1}(y, x) \ni z .
\end{aligned}
$$


So, $\left(Q ;^{-1} A\right)$ is $a$ hypercancellation hypergroupoid of the type $\langle q, p, r\rangle$.

Theorem 4.2. Let $(Q ; A)$ be a finite hypergroupoid with divisions. Then $(Q ; A)$ is a hypercancellation hypergroupoid.

Proof. By Def. 1.1., Def. 3.1, Def. 4.1 and by $|Q| \in N$.

Theorem 4.3. There is a hypergroupoid $(Q ; A)$ such that the following statements hold:

a) $(Q, A)$ is a hypergroup; and

b) $(Q ; A)$ is not a hypercancellation hypergroupoid.

Proof. Let $(Q ; \cdot)$ be an infinite group. Also, let

$$
A(x, y) \stackrel{\text { def }}{=}\{x \cdot k \cdot y|k \in \bar{Q} \wedge \bar{Q} \subseteq Q \wedge| \bar{Q}|\geq| N \mid\}
$$

for all $x, y \in Q$. Then $(Q ; A)$ is a hypergroup. Moreover, $(Q ; A)$ is not a hypercancellation hypergroupoid.

\section{Hyperquasigroups}

Definition 5.1. We shall say that a hypergroupoid $(Q ; A)$ is a hyperquasigroup iff $(Q ; A)$ is a hypergroupoid with divisions and hypercancelation hypergroupoid as well ${ }^{4}$.

Remark 5.1. Since each hyperquasigroup $(Q ; A)$ is a hypercancellation hypergroupoid of the type $\langle p, q, r\rangle$, we shall say that it is a hyperquasigroup of the type $\langle p, q, r\rangle$. Moreover, we called $m$ is a rang hyperquasigroup $(Q ; A)$ of the type $\langle p, q, r>$ iff $m=\max \{p, q, r\}$. Together, by Th. 4.4, for all $\alpha \in\{1,2,3\}$ ! $\operatorname{rang}\left(Q ; A^{\alpha}\right)=m$.

Remark 5.2. Groupoid $(Z ; \mathcal{A})$ from Example 4.3 is a groupoid with divisions. However, $(Z, \mathcal{A})$ is not a cancelation groupoid, i.e. $(Z ; \mathcal{A})$ is not a quasigroup.

By Th. 4.5 and by Def. 5.1, we obtain:

Theorem 5.1. Every finite hypergroupoid with divisions is a hyperquasigroup.

By Th. 4.6, Def. 1.4 and by Def. 5.1, we have:

Theorem 5.2. There are hypergroups with are not hyperquasigroups.

By Def. 1.4, Def. 3.1 and by Th. 5.4, we obtain:

Theorem 5.3. Every finite hypergroup is a hyperquasigroup.

\footnotetext{
${ }^{4}$ See, also [3]
} 


\section{6. $3-<m>-\mathrm{NET}$}

Definition 6.1. Let $\Im$ be a non-empty set and let $\mathcal{L}$ be a non-empty subset of the set $\Im$. We shall say that the elements of $\Im$ are points and the elements of $\mathcal{L}$ are lines. Also, let $\left\{L_{1}, L_{2}, L_{3}\right\} \stackrel{\text { def }}{=} \mathcal{L} / \sim$, where $\sim$ is a equivalence relation on the set $\mathcal{L}$. Then, we shall say that the object $\left(\Im ; L_{1}, L_{2}, L_{3}\right)$ is a $3-<m>$-net, $m \in N$, iff the following conditions hold:

M1. Each point belongs to exactly one line of each equaivalence (paralel) class $\left[L_{1}, L_{2}\right.$ and $\left.L_{3}\right]$.

M2 1 . If $L_{a}$ and $L_{b}$ are different classes of $\left\{L_{1}, L_{2}, L_{3}\right\}$, then for all $l_{1} \in L_{a}$ and $l_{2} \in L_{b}$ the following formula holds $\left|l_{1} \cap l_{2}\right| \leq m$.

M2 2 . If $L_{a}$ and $L_{b}$ are different classes of $\left\{L_{1}, L_{2}, L_{3}\right\}$, then for all $l_{1} \in L_{a}$ and $l_{2} \in L_{b}$ the following formula holds $\left|l_{1} \cap l_{2}\right| \geq 1$.

M23. There is at least one $\left(l_{1}, l_{2}\right) \in L_{a} \times L_{b}$, where $a, b \in\{1,2,3\}$ and $a \neq b$, such that the following equality holds $\left|l_{1} \cap l_{2}\right|=m$.

M3. $\left|L_{1}\right|=\left|L_{2}\right|=\left|L_{3}\right|$.

M4. Let $L_{a}, L_{b}$ and $L_{c}$ be arbitrary mutually different classes of $\left\{L_{1}, L_{2}, L_{3}\right\}$. Then, for all $A, B \in \Im$ and for every $l_{1} \in L_{a}, l_{2} \in L_{b}$ and $l, \bar{l} \in L_{c}$ the following condition holds

$$
A \neq B \wedge A \in l_{1} \cap l_{2} \wedge B \in l_{1} \cap l_{2} \wedge A \in l \wedge B \in \bar{l} \Rightarrow l \neq \bar{l}^{5}
$$

Remark 6.1. $3-<1>-$ net is a 3 -net. Cf. $[4,5,6]$.

Remark 6.2. $\quad$ a) The conditions M1-M4 (for $m=2$ ) hold in the object represented to Diagram 1.

b) The conditions M1-M3 (for $m=2$ ) hold in the object represented to Diagram 2. However, condition M4 does not hold.

\section{HyPERQUASIGROUPS AND $3-<m>-$ NETS}

Theorem 7.1. Let $(Q ; A)$ be a hyperquasigroup, $|Q| \geq 2$ and let $\operatorname{Rang}(Q ; A)=$ $m[\in N]$. Also, let

$(t) \Im \stackrel{\text { def }}{=}\{(x, y, z) \mid x \in Q \wedge y \in Q \wedge A(x, y) \ni z\}$,

(c $\left.c_{1}\right) L_{1} \stackrel{\text { def }}{=}\left\{(x, y, z)\left|y \in Q \wedge z \in Q \wedge^{-1} A(z, y) \ni x\right| x \in Q\right\}$

$\left(c_{2}\right) L_{2} \stackrel{\text { def }}{=}\left\{(x, y, z)\left|x \in Q \wedge z \in Q \wedge A^{-1}(x, z) \ni y\right| y \in Q\right\}$ and

$\left(c_{3}\right) L_{3} \stackrel{\text { def }}{=}\{(x, y, z)|x \in Q \wedge y \in Q \wedge A(x, y) \ni z| z \in Q\}$.

Then $\left(\Im ; L_{1}, L_{2}, L_{3}\right)$ is a $3-<m>-$ net.

Proof. 1) By $\left(c_{1}\right)-\left(c_{3}\right)$ and by Def. 5.1, we have: $L_{1} \cap L_{2}=L_{1} \cap L_{3}=$ $L_{2} \cap L_{3}=\emptyset$ and $\left|L_{1}\right|=\left|L_{2}\right|=\left|L_{3}\right|=|Q|$ [M3].

2) By $(t),\left(c_{1}\right)-\left(c_{3}\right)$ and by Def. 5.1, we obtain M1.

3) By $\operatorname{Rang}(Q ; A)=m$, Rem. 5.2 and by $\left(c_{1}\right)-\left(c_{3},\right)$ we have $\mathbf{M} 2_{1}$ and $\mathrm{M2}$.

4) By $\left(c_{1}\right)-\left(c_{3}\right)$ and by Def. 5.1 [Def. 3.1], we obtain M2 2 . 
4) By $\quad\left(x, y, z_{1}\right) \neq\left(x, y, z_{2}\right) \Longleftrightarrow z_{1} \neq z_{2}$,

$$
\begin{aligned}
& \left(x, y_{1}, z\right) \neq\left(x, y_{2}, z\right) \Longleftrightarrow y_{1} \neq y_{2} \text { and } \\
& \left(x_{1}, y, z\right) \neq\left(x_{2}, y, z\right) \Longleftrightarrow x_{1} \neq x_{2},
\end{aligned}
$$

we have, also, M4.

Remark: For $m=1$ see Rem. 6.2.

Theorem 7.2. Let $\left(\Im ; L_{1}, L_{2}, L_{3}\right)$ be a $3-<m>-$ net. Also let

$\left(l_{1}\right) L_{1}=\{<1, x>\mid x \in Q\}$,

$\left(l_{2}\right) L_{2}=\{<2, x>\mid x \in Q\}$ and

$\left(l_{3}\right) L_{3}=\{<3, x>\mid x \in Q\}$,

where $Q$ is an arbitrary set such that $|Q|=\left|L_{1}\right|\left(=\left|L_{2}\right|=\left|L_{3}\right|\right)$. Finally, let

(h) $A(x, y) \ni z \stackrel{\text { def }}{\Leftrightarrow}(\exists T \in \Im) T \in<1, x>\cap<2, y>\cap<3, z\rangle$

for all $x, y, z \in Q$. Then, $(Q ; A)$ is a hyperquasigroup of the rang $m$.

Proof. $\quad 1)$ By $\mathbf{M 1}$ and by $(h)$, we conclude that $A$ is a hyperoperation in $Q$.

2) By $\mathbf{M} \mathbf{2}_{2}, \mathbf{M} 3$ and by 1$)$, we have $(Q ; A)$ is a hypergroupoid with divisions.

3) By $\mathbf{M} 2_{1}, \mathbf{M 2}_{3}$, by Def.4.1 and by 1$)$, we conclude that $(Q ; A)$ is a hypercancelative hypergroupoid.

4) By $\left.\left.\mathbf{M} \mathbf{2}_{1}, \mathbf{M} \mathbf{2}_{3}, 2\right), 3\right)$, Def. 5.1 and by Rem. 5.2, we conclude that $(Q ; A)$ is a hyperquasigroup of the rang $m$.

Remark: By M4 and M1, $F: \Im \rightarrow\{(x, y, z) \mid(h)\}$ is a bijection. See, also Rem. 6.3, Th.7.1 and Th.7.2.

Theorem 7.3. Let $(Q ; A)$ be a hyperquasigroup. Then, there are permutations $\alpha, \beta, \gamma$ of $Q$ such that the following formula holds

$$
(\exists e \in Q)(\exists x \in Q)(L(e, x) \ni x \wedge L(x, e) \ni x),
$$

where

$$
L(x, y) \stackrel{\text { def }}{=} \gamma A(\alpha(x), \beta(y))
$$

and

$$
\varphi\left\{a_{1}, \ldots, a_{s}\right\} \stackrel{\text { def }}{=}\left\{\varphi\left(a_{1}\right), \ldots, \varphi\left(a_{s}\right)\right\}, \quad a_{1}^{s} \in Q, \quad \varphi \in Q !, \quad 1 \leq s \leq m .{ }^{6}
$$

Proof. By Def.6.1, Th. 7.1 and by Th. 7.2. See Diag. 3;

$$
1 \leq|<1, e>\cap<2, x>| \leq m, \quad 1 \leq|<3, x>\cap<2, e>| \leq m .
$$

See, also [5], p.p. $13-16$.

\section{REMARKS}

Remark 8.1. Hypergroupoid $(Q ; A)$ defined by Table 3 is a hyperquasigroup. Hypergroupoid $(Q ; \bar{A})$, where $\bar{A}(x, y)=A(x, y)$ for all $(x, y) \in Q^{2} \backslash\{(3,3)\}$ and $\bar{A}(3,3)=\{1\}[\bar{A}(3,3) \subseteq A(3,3) \wedge \bar{A}(3,3) \neq A(3,3)]$, is too a hyperquasigroup.

Remark 8.2. Hipergroupoid defined by Table 4 is a hyperquasigroup. For all $(Q ; \bar{B})$, where $\bar{B}(x, y)=B(x, y)$ for all $(x, y) \in Q^{2} \backslash\{(1,3),(3,2)\}, \bar{B}(1,3) \subseteq$ $B(1,3), \bar{B}(3,2) \subseteq B(3,2)$ and $|\bar{B}(1,3)|=|\bar{B}(3,2)|=1$, is not a hyperquasigroup. 


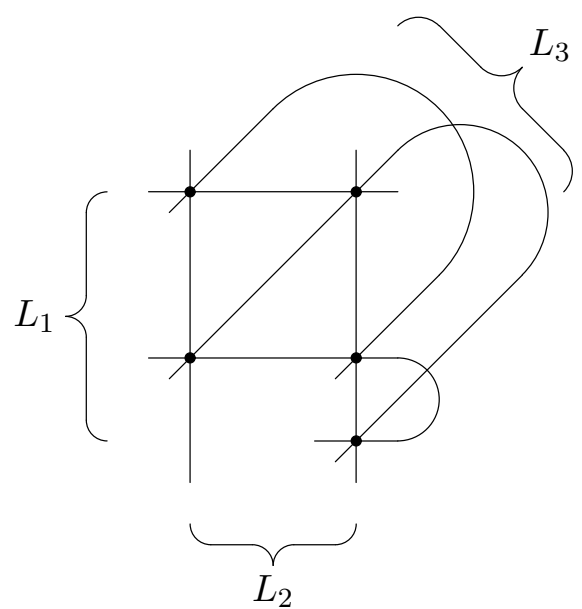

Diagram 1.

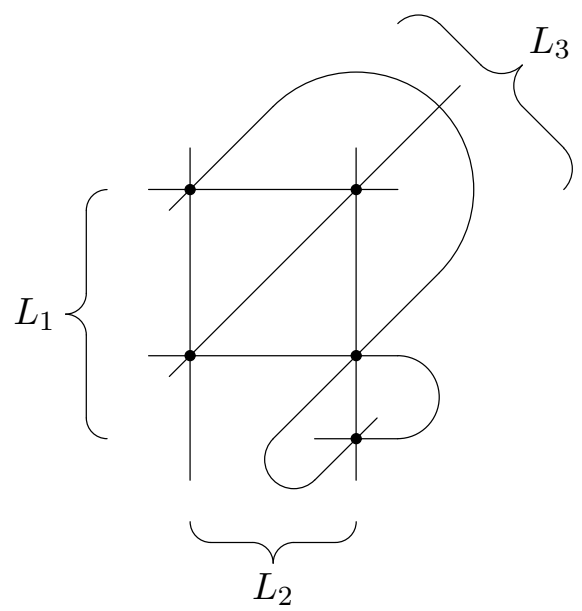

Diagram 2.

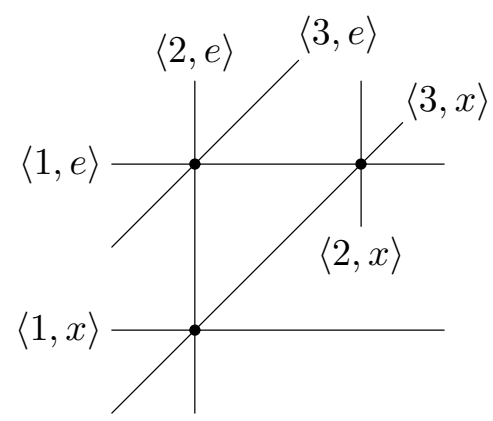

Diagram 3. 


\begin{tabular}{c|c|c|c}
$A$ & 1 & 2 & 3 \\
\hline 1 & $\{1\}$ & $\{2\}$ & $\{3\}$ \\
\hline 2 & $\{3\}$ & $\{1\}$ & $\{2\}$ \\
\hline 3 & $\{2\}$ & $\{3\}$ & $\{1,2\}$ \\
\hline
\end{tabular}

Table 3

\begin{tabular}{c|c|c|c}
$B$ & 1 & 2 & 3 \\
\hline 1 & $\{1\}$ & $\{1\}$ & $\{2,3\}$ \\
\hline 2 & $\{2\}$ & $\{1\}$ & $\{3\}$ \\
\hline 3 & $\{3\}$ & $\{2,3\}$ & $\{1\}$ \\
\hline
\end{tabular}

Table 4

Remark 8.3. In the searching for notion of hyperquasigroup there has been in aspect, also, the following:

1) There is groupoid with divisions which is not a quasigroup; and

2) Every semigroup with divisions is a quasigroup (group). [We say that a groupoid $(Q ; \cdot)$ is a groupoid with divisions iff for every $a, b \in Q$ there exist at least one $x \in Q$ and at least one $y \in Q$ such that the following equalities hold $a \cdot x=b$ and $y \cdot a=b$.]

\section{REFERENCES}

[1] F. Marty, Sur une généralization de la notion de groupe, Huitiéme congres de mathématiciens Scandinaves, Stockholm, (1934), 45-49.

[2] J. Mittas, Hypergroupes canoniques valués et hypervalués, Math. Balkanica 1(1971), 181-185.

[3] G. Tallini, Geometric Hyperquasigroups and Line Spaces, Acta Universitatis Carolinae Mathematica et Physica, Vol. 25, No. 1(1984), 69-73.

[4] V. D. Belousov, Foundation of the theory of quasigroups and loops, (Russian), "Nauka", Moscow 1967.

[5] V. D. Belousov, Algebraic nets and quasigroups, (Russian), Stiinta, Kishinev 1971.

[6] J. Dénes and A. D. Keedwell, Latin Squares - New Developments in the Theory and Applications, North-Holland 1991.

[7] J. Ušan, $k-<2>-n e t s$, (Russian), Rev. of Research, Fac. of Sci. Univ. of Novi Sad, Math. Ser., 16, 2(1986), 173-196.

Institute of Mathematics
University OF Novi SAD
Trg D. OBradovića 4
21000 Novi SAd,
SERBia AND MonteneGro

FACUlty of Electrical EngineERing UNIVERSITY OF OSIJEK

KNEZA TRPIMIRA 2B

HR - 31000 OSIJEK

CROATIA 